FORMATION Formation emploi

Revue française de sciences sociales

108 | octobre-décembre 2009

Les processus de professionnalisation

\title{
Être salarié dans un club sportif : une posture problématique
}

Ser asalariado en un club deportivo : una postura problematica

To be an employee of a sports club: a difficult position

Angestellter im Sportverein: ein problematischer Status

\section{Marc Falcoz et Emmanuelle Walter}

\section{(2) OpenEdition}

Journals

\section{Édition électronique}

URL : http://journals.openedition.org/formationemploi/2067

DOI : 10.4000/formationemploi.2067

ISSN : 2107-0946

\section{Éditeur}

La Documentation française

\section{Édition imprimée}

Date de publication : 1 décembre 2009

Pagination : 25-37

ISSN : 0759-6340

\section{Référence électronique}

Marc Falcoz et Emmanuelle Walter, "Être salarié dans un club sportif : une posture problématique », Formation emploi [En ligne], 108 | octobre-décembre 2009, mis en ligne le 01 janvier 2012, consulté le 10 décembre 2020. URL : http://journals.openedition.org/formationemploi/2067 ; DOI : https:// doi.org/10.4000/formationemploi.2067 


\title{
DOSSIER
}

\section{Être salarié dans un club sportif: une posture problématique}

\author{
Par Marc Falcoz et Emmanuelle Walter *
}

\section{Recrutés sur la base de leurs qualifications professionnelles, les salariés du sport associatif français se trouvent pourtant dans une posture problématique, leur reconnaissance par les dirigeants bénévoles passant principalement par l'engagement et le don de soi.}

Cet article vise à montrer en quoi le travail salarié dans les clubs sportifs français s'inscrit dans une posture problématique particulière. Le cas du travail associatif sportif se positionne certes dans un contexte plus général d'institutionnalisation de l'emploi salarié dans le secteur associatif et s'inscrit dans le cadre de travaux qui montrent l'ambivalence d'un salariat « non ordinaire » ( $c f$. encadré $\mathbf{1})$.

Mais le champ sportif révèle un certain nombre de spécificités qui caractérisent de manière particulière les acteurs salariés des clubs sportifs : des causes structurelles spécifiques peuvent d'abord être mobilisées pour expliquer les limites du développement des formes d'emploi dans ce secteur. Ensuite, la persistance d'un taux très élevé de bénévoles dans l'encadrement sportif montre que la question de l'emploi dans les associations sportives est indissociable de celle du bénévolat. Si le développement du travail rémunéré dans les clubs sportifs est une réalité, les associations sportives restent dirigées par des bénévoles toujours très présents dans cet espace associatif. De fait, les acteurs salariés du sport associatif (entraîneurs, moniteurs, éducateurs, agents et cadres
* Marc Falcoz est maître de conférences. II fait partie du Laboratoire d'étude et de recherche sur les professionnalisations (LERP/ JE 2537), UFR-STAPS (Sciences et techniques des activités physiques et sportives), université de Reims Champagne-Ardenne. Appartenance disciplinaire: 74e section (Sciences et Techniques des Activités Physiques et Sportives).

Ses domaines de recherches sont les organisations sportives, la professionnalisation dans le secteur sportif.

Emmanuelle Walter est maître de conférences. Elle fait partie du LERP. Appartenance disciplinaire : 74e section (Sciences et Techniques des Activités Physiques et Sportives).

Ses domaines de recherche sont le bénévolat, les associations sportives, la professionnalisation dans les clubs sportifs.

Ils ont publié conjointement: Falcoz M., Walter E. (2007), "Travailler dans un monde de bénévoles: contraintes et limites de la professionnalisation dans les clubs sportifs ", Revue des études coopératives, mutualistes et associatives, RECMA, $n^{\circ}$ 306, pp. 78-91. Falcoz M., Walter E. (2009), "L'emploi dans le sport associatif et .../... 
fédéral. Un état de la question », Revue internationale des Sciences du Sport et de l'Education Physique. STAPS, $n^{\circ} 83$, pp. 43-54.

administratifs) sont placés dans un contexte qui les soumet à une injonction ambivalente : d'un côté, ils sont recrutés au regard de compétences professionnelles spécifiques (attestées par la possession d'un diplôme) et sont tenus d'assurer les fonctions pour lesquelles ils sont rémunérées. Mais d'un autre côté, les attentes de leurs employeurs (bénévoles) se focalisent sur les capacités de l'employé à faire la preuve d'un savoir-être en termes de disponibilité, de don de soi (de son temps, de sa personne), et d'engagement

\section{Encadré 1 \\ Positionnement théorique}

Les travaux précurseurs d'E. Marchal (1992), puis ceux de M. Hely (2004, 2008) permettent de penser les milieux associatifs comme des mondes du travail. En objectivant le développement de formes diverses "d'entreprises associatives", ces auteurs analysent les effets d'un processus de "salarisation " sur les structures et les acteurs du secteur associatif. Mais si la progression de l'emploi dans le secteur associatif est indéniable $\left({ }^{*}\right)$, les salariés évoluent dans un contexte institutionnel qui demeure marqué par les logiques du bénévolat et du militantisme. Ainsi dans ce type d'organisation, la structure du lien social croise les logiques du don, incarnées par le bénévolat, et celles du contrat, incarnées par le salariat (Hely, 2004). De même, dans ses travaux sur les associations intermédiaires, A. Legay (2001) révèle aussi le caractère atypique de ce salariat, tendu entre "le souci de rendre un service de qualité » et celui de "préserver la posture militante ". La prégnance des logiques de l'engagement s'exerce donc sur la construction de "professionnalités particulières » (Legay, 2001).

Le cas des associations sportives s'inscrit dans ce contexte général, mais revêt des spécificités liées au domaine sportif. Si ce dernier présente effectivement un terrain où les formes d'emploi se développent significativement (Leroux, Aguettant, 2006), le volume de l'emploi y reste bien inférieur à celui du bénévolat dont l'encadrement demeure une activité ultra-légitime. Loin de se développer dans toutes les formes de l'encadrement associatif et dans tous les types d'associations sportives, I'emploi sportif associatif se concentre surtout sur les métiers de l'encadrement technique (entraîneurs, animateurs sportifs) et dans le secteur du sport associatif compétitif fédéré (Loirand, 2003). Dans ce contexte, parler de "professionnalisation » dans les associations sportives semble relever davantage de propos performatifs que d'une analyse véritablement objective (Loirand, 2004). Les freins au développement de l'emploi dans les associations sportives peuvent certes être reliés à des causes structurelles lémiettement du champ associatif sportif, petites tailles des structures, importance du caractère saisonnier et précaire du travail sportif, surinvestissement de l'emploi aidél) et à la spécificité d'un champ sportif où le " culte du résultat » et les "passions sportives" présupposent une acceptation naturelle de la précarité inhérente aux aléas de la compétition (Fleuriel, Schotté, 2008). Mais si les effets spécifiques de ce champ sportif agissent autant sur les acteurs, c'est surtout parce qu'ils sont prédisposés par leurs trajectoires à " entrer dans le jeu »; ainsi le capital sportif peut être défini comme une sorte de capital culturel, à la fois socialisé et incorporé, et dont l'acquisition se révèle nécessaire pour exister dans le champ sportif (Bourdieu, 1998). Tendu entre le souci de représenter l'idéal de désintéressement et d'amateurisme cher au Mouvement sportif, et celui de faire valoir des compétences professionnelles traversées notamment par une valeur sportive convertible en valeur économique, ce capital sportif se structure de fait au contact d'un champ sportif lui-même ambivalent. 
au service du club sportif. La mobilisation des apports du structuralisme critique ${ }^{1}$ se révèle ici féconde pour mettre en relation les caractéristiques du champ sportif et celles du capital sportif nécessaire pour exister dans cet espace. Plus précisément, l'analyse des trajectoires des salariés du secteur met en exergue les dispositions acquises nécessaires pour exister dans un espace à la fois soumis à la " passion du sport » et à la prégnance des valeurs et de l'idéologie bénévole sportive ( $c f$. encadré 1). Ce travail s'appuie sur un corpus d'enquêtes et d'interviews menés auprès d'un échantillon d'associations sportives employeurs de la Marne (51) et de leurs acteurs salariés et bénévoles ( $c f$. encadré 2).

Ainsi nous montrerons d'abord en quoi le monde sportif associatif présente la particularité d'être à la fois marqué par une progression significative de l'emploi salarié et par une persistance très forte de la gouvernance bénévole. Confrontées à la concurrence d'autres formes d'offre en pratiques d'exercice corporel (secteur marchand et public), les associations sportives recrutent effectivement un nombre croissant de salariés pour répondre à une demande plus forte en encadrement qualifié (Loirand, 2003) et à un souci de rationalisation managériale de leur offre (Chantelat, 2001). Mais si ce développement économique s'inscrit dans un contexte général de "salarisation » de l'activité associative (Hely, 2004, 2008), il se révèle étonnamment modeste dans le secteur associatif sportif, où l'association demeure effectivement le refuge des valeurs du sport dites originelles; car « amateurisme », « désintéressement» et "gratuité du geste sportif » constituent le socle historique d'un Mouvement sportif toujours convaincu d'être le gardien des « vraies » valeurs du sport.

Nous montrerons ensuite que forts de leur légitimité morale liée à ce contexte, les bénévoles occupent une position ultra dominante dans le champ sportif, quoique ambivalente. Comment se positionnent-ils et comment leur «double posture» produit-elle des exigences contradictoires qui s'imposent à leurs employés salariés?

${ }^{1}$ Comme analyse du rapport entre les positions sociales (concept relationnel), les dispositions (ou les habitus) et les prises de position, les « choix » que les agents sociaux opèrent dans les domaines les plus différents de la pratique (Bourdieu, 1996).
Enfin, nous analyserons la posture de ces mêmes acteurs salariés, et notamment les rapports entre leurs dispositions sociales, leurs trajectoires et les représentations relatives à leurs conditions « professionnelles». En quoi la prégnance du champ sportif et de ses spécificités s'impose-t-elle aux salariés et les structure-t-elle dans une professionnalité problématique?

\section{Encadré 2 \\ L'enquête de terrain}

Notre analyse s'appuie conjointement sur l'étude structurelle de 18 clubs sportifs marnais et sur l'analyse du discours de 45 acteurs de ces clubs 118 bénévoles et 27 salariés). Sur la base du fichier SIRENE(*) des 255 associations employeurs de la Marne classées sous le code NAF (Nomenclature d'activités française, révision 1, 2003) 92.6 (Services liés au sport) les associations sportives sélectionnées font parties des trois disciplines sportives présentant le plus grand nombre d'associations employeurs et dont le développement effectif de l'emploi salarié demeure le plus patent, à savoir le tennis, le football et les activités gymniques. Puis nous avons différencié, au sein de cette base de clubs sportifs, le groupe des "petites associations sportives employeurs " (deux salariés et moins / $n=9$ ), et le groupe des "grandes associations sportives employeurs" (trois salariés et plus / $n=9$ ). Dans le premier groupe, nous avons interviewé systématiquement un dirigeant bénévole et un cadre salarié, et dans le second un dirigeant bénévole et deux cadres salariés.

$\left.{ }^{*}\right)$ : Le répertoire SIRENE « Système Informatique pour le Répertoire des Entreprises et de leurs Établissements », créé en 1973, constitue l'état civil de toutes les entreprises et établissements français. Il propose, dans un cadre commercial, des bases de données d'entreprises ou d'établissements avec l'adresse, des caractéristiques économiques de chaque unité. Géré par l'INSEE (Institut national de la statistique et des études économiques), il attribue un numéro de neuf chiffres à toute personne, qu'elle soit physique ou morale, qui exerce une activité professionnelle. 


\section{LES PARADOXES DE L'IDENTITÉ ASSOCIATIVE SPORTIVE}

\section{De la progression de l'emploi salarié dans les clubs sportifs...}

Les associations sportives sont effectivement traversées, à l'instar de l'ensemble du secteur associatif, par un accroissement du volume de l'emploi salarié au niveau de l'encadrement technique et administratif (Tchernonog, 2007). Certes, la mesure objective de ce processus souffre de la dispersion des activités relatives au sport dans les nomenclatures d'activités économiques et de professions (Le Roux, Aguettant, 2006)2.

Mais la conjonction d'un nombre croissant de travaux atteste de la pénétration de l'emploi dans un espace historiquement constitué autour du bénévolat. Malgré les difficultés de nomenclature finement analysées par N. Le Roux et N. Aguettant (2006), ces mêmes chercheurs estiment à près de 100000 le nombre de salariés de droit privé qui sont employés dans le secteur du sport en 2003 (hors indépendants $)^{3}$; ce qui représente un taux de croissance annuel moyen des effectifs salariés de 5,9\% sur la période 1994-2003. D'après les travaux du RUNOPES (Réseau universitaire d'observation et de prospective de l'emploi sportif), les structures associatives profitent largement de cette professionnalisation effective. Dans le sous-secteur des loisirs sportifs comprenant notamment les gymnastiques de

\footnotetext{
${ }^{2}$ L'activité économique liée au sport peut être considérée comme un secteur d'activité regroupant des activités très diverses nécessaires ou induites par le développement de la pratique sportive. On peut en distinguer deux grands groupes. Le premier groupe, le «secteur sport», se limite aux activités de services qui sont en relation directe avec la pratique sportive et en représentent le noyau dur, comme par exemple les activités d'animation et d'encadrement technique de la pratique sportive. Le second groupe, la " filière sport et les secteurs associés », correspond aux activités économiques en relation avec le sport comme par exemple les métiers de la santé (diététique, médecine du sport...), du commerce d'articles de sport (vente et marketing du sport) et de la communication (journalisme sportif par exemple) Dans les deux groupes, on rencontre de multiples difficultés techniques et théoriques pour identifier les emplois que l'on peut considérer comme « sportifs »; ce qui accroît les problèmes de lisibilité de la professionnalisation dans le sport (Le Roux, Aguettant, 2006).

${ }^{3}$ Chiffre obtenu grâce aux PSB (Portraits statistiques de branche), réalisés et mis à disposition par le Céreq à partir de multiples sources de statistiques publiques.
}

loisir associatives, les activités nautiques et aquatiques et les loisirs de raquette (surtout le tennis), la majorité des structures sont des associations (soient 22000 emplois environ pour les trois catégories citées). Concernant le secteur du sport associatif compétitif fédéré, qui représente $30 \%$ des structures employeurs du secteur sport (Le Roux, Aguettant, 2006), on est ainsi passé de 16649 employés sportifs comptabilisés dans l'Enquête Emploi de 1982 à 66658 employés sportifs en 1999 (Loirand, 2003).

Plusieurs raisons, sans prétendre à l'exhaustivité, peuvent être évoquées pour expliquer le développement du volume de l'emploi salarié dans les clubs sportifs.

En premier lieu, soulignons l'importance des dispositifs d'aide à l'emploi mis en place au cours des vingt dernières années, et auxquels les associations sportives ont répondu très favorablement. Dans le cadre de la lutte contre le chômage, l'État s'est engagé, à travers différents plans pour l'emploi (plan sport emploi, emploi jeune, contrat d'avenir, parcours animation sport...), à faciliter la structuration de l'encadrement et l'animation des associations sportives. Les clubs sportifs ont ainsi été considérés « comme des structures favorables à la réinsertion des jeunes en perte de repères » (Le Roux, 2006), et plus de 52000 postes d'emplois jeunes dans le secteur associatif « jeunesse et sport», dont près de 17000 dans des associations sportives, ont été créés par le programme «nouveaux services, nouveaux emplois ».

En deuxième lieu, selon Gildas Loirand (2003, 2004), le développement de l'emploi technique (entraîneur en particulier) dans le sport associatif compétitif fédéré est lié à l'accroissement de la pression compétitive au niveau amateur dans de nombreux domaines sportifs. Les valeurs et le modèle du sport de haut niveau exercent un effet de séduction sur les dirigeants associatifs de clubs même modestes, et entrainent un besoin croissant en compétences qualifiées au niveau de l'encadrement technique. Dans le cadre de nos enquêtes, ce constat est effectivement confirmé dans les domaines sportifs du tennis et du football au niveau de clubs représentant une forme d'élite sportive locale et/ou régionale. $\mathrm{La}$ recherche des résultats sportifs est 
présentée par tous les dirigeants interviewés comme l'objectif premier du club. Il apparait clairement que le développement de la compétitivité sportive du club demeure au cœur de l'identité associative, car elle garantit le maintien, voire la progression de l'association dans la hiérarchie sportive régionale ou nationale.

En troisième lieu, la pénétration de l'emploi dans les associations sportives peut aussi être reliée aux transformations de l'offre et de la demande sportive en général. Ces dernières obligent les clubs et les fédérations à réagir pour répondre aux nouvelles aspirations sportives mais aussi au développement de la concurrence de l'offre privée marchande et de l'offre publique sur le marché des prestations sportives. En effet, le temps de l'hégémonie fédérale et associative est bien révolu, et le système d'offre en pratiques sportives et corporelles est désormais un marché organisé par une concurrence qui s'accroît entre les différentes structures d'offre (Augustin, 2003). C'est aussi ce que renvoie Pascal Chantelat (2001, p. 15), quand il souligne que " le monde associatif est investi par un ensemble de discours et de pratiques managériales qui visent à rationaliser le fonctionnement des organisations et à optimiser leurs performances pour répondre aux besoins des pratiquants sportifs. Cette rationalisation passe, notamment, par le recrutement de salariés et de bénévoles compétents ».

En effet, les activités d'éveil basées sur la gymnastique et proposées au tout jeune enfant constituent souvent une première activité sportive fortement prisée par les parents.

En conséquence, même si pour la plupart des enfants ces activités ne se prolongent pas vers une formation sportive et compétitive en gymnastique, elles deviennent structurantes de la vie associative de ces clubs en créant de l'emploi pour répondre à une forte demande en encadrement professionnel et qualifié. Ce sont bien les activités de « loisir » intégrées dans l'offre associative qui constituent à présent l'ossature du club, laquelle a «glissé » du tout bénévolat vers une structuration à base professionnelle, principe de sa nouvelle attractivité; les emplois créés dans ce contexte nouveau portent la marque de ces évolutions et permettent une refondation des activités gymniques sur d'autres bases et en relation avec une demande nouvelle. «On peut observer une demande de plus en plus importante au niveau des membres pour avoir des professionnels qui encadrent leurs enfants... au début, il y a énormément de bénévoles et on sentait souvent les parents réticents, même si les bénévoles avaient des diplômes fédéraux, de voir des jeunes encadrer leurs enfants (....) donc c'est pour ça qu'en 15 ans de temps, il y a eu une forte professionnalisation du club au niveau de l'encadrement, mais c'était plus, on sentait plus une demande des membres » (Présidente du club de gymnastique M) ${ }^{4}$.

\section{... à la domination du bénévolat}

Mais si le processus de développement de l'emploi salarié ne peut pas être nié, nous pensons qu'il ne remet pas en cause la structuration fondamentale et historiquement constituée du club sportif autour de l'encadrement et des valeurs bénévoles.

En premier lieu, nous pouvons rappeler, avec Gildas Loirand, (2004) que le terme même de " professionnalisation » prête à confusion et sert à désigner trop hâtivement un passage de l'amateurisme au professionnalisme relevant plus du discours que de la réalité. Autrement dit, il ne faut pas confondre une augmentation, même significative, du volume d'emplois dans les associations sportives, avec une logique de « professionnalisation » du secteur.

En second lieu, plusieurs travaux révèlent la faiblesse structurelle de l'emploi dans les associations sportives; une enquête (Ithaque, 2006) menée à l'initiative de l'Observatoire des métiers de l'animation et du sport (OMNAS) auprès de 2845 entreprises relevant de la convention collective du sport (CS) et adhérentes aux collecteurs de la formation professionnelle (Uniformation et Agefosi PME) montre que le secteur de l'emploi associatif sportif est principalement composé de petites structures employant avant tout à temps partiel. En effet, les entreprises avec seulement un salarié représentent $41 \%$ du total des employeurs de la branche et $37 \%$ des entreprises ont moins d'un emploi équivalent temps plein. Même si l'enquête conclut que les entreprises prévoient, à $42 \%$, des besoins de recrutement croissants dans

${ }^{4}$ Afin de respecter l'anonymat des personnes interviewées, leurs clubs d'appartenance sont identifiés de la manière suivante: tennis : A à $\mathrm{F} /$ football : $\mathrm{G}$ à $\mathrm{L} / \mathrm{gym}$ forme et loisir : $\mathrm{M}$ à $\mathrm{R}$. 
l'avenir, la situation de l'emploi associatif sportif demeure fragile. Ce constat est corroboré par les travaux de N. Le Roux (2006) quand elle souligne que le secteur sportif «cumule toutes les caractéristiques d'un secteur où règne la précarité $» .5$

En troisième lieu, le monde sportif se targue d'avoir su rester, au niveau de ses associations sportives au moins, un espace privilégié de l'engagement « gratuit» et des valeurs d'un amateurisme distinctif. En effet, l'organisation associative du sport en France prend tout d'abord racine dans l'idée associative elle-même. Dès ses origines, elle incarne des valeurs qui ne se veulent pas dominées par le souci du profit individuel et de l'enrichissement personnel, mais par celui de l'intérêt général, de la citoyenneté, du partage des richesses, de la cohésion sociale (Chanial, 1998). Les pratiques bénévoles sont représentatives de ces valeurs car le bénévolat réhabilite la notion de gratuité et de « désintéressement » économique à travers l'expression d'un altruisme et d'une «bienveillance» inscrite dans sa définition même. Dans cet univers hautement symbolique qu'est le sport, le bénévolat est fréquemment convoqué dans les discours officiels (notamment des dirigeants du sport fédéral et olympique national et international) pour rappeler "l'essence» universelle du sport (Liotard, 2004). En contrepoint, le travail et les professionnels du sport apparaissent comme une altération de la posture légitime et historique du sport, et ce d'autant plus qu'ils peuvent être aisément accusés d'avoir introduit dans cet espace originellement «pur» les perversions liées à l'argent et à ses dérives (Fleuriel, 2006). Cette opposition bénévolattravail rémunéré, bien que déniée, reste une caractéristique structurante d'un monde sportif travaillé par ses mythes et par l'idée d'une " exception sportive » bien particulière (Falcoz, Walter, 2007).

\footnotetext{
${ }^{5}$ Les temps partiels et CDD (contrat à durée déterminée) caractérisent toujours un grand nombre d'emplois du secteur. La part des emplois salariés en contrat à durée déterminée a crû notablement au cours de ces dernières années, jusqu'à représenter $70 \%$ des entrées dans les établissements de 10 salariés ou plus du secteur (Le Roux, 2006).
}

\section{LES DIRIGEANTS BÉNÉVOLES EN POSITION DE FORCE}

Dans un tel contexte, les dirigeants bénévoles sont tendus entre un principe "pragmatique» (qui consiste à accepter une certaine professionnalisation comme nécessaire à la modernisation de l'encadrement sportif) et un principe « éthique » de conservation des valeurs historiques qui font l'identité de la cause associative sportive.

D'un côté, ils doivent montrer qu'ils sont capables de saisir les enjeux en cours et d'être des entrepreneurs de la modernisation de l'offre associative sportive. Ils ne peuvent donc pas refuser le développement de l'emploi dans les clubs sportifs sous peine d'être durablement concurrencés par le secteur privé marchand et de se rendre responsables de l'inadaptation croissante du secteur associatif sportif aux évolutions de la demande sportive. "Le club a suivi ces évolutions parce qu'il y a une nécessité à un moment donné de s'adapter aux conditions socioéconomiques d'un environnement donné. On est obligé de faire ça, parce que sinon on ne va pas survivre, parce que financièrement on ne va pas s'inclure dans le monde socio-économique» (Président du club de gymnastique $\mathrm{O}$ ). Mais présentée comme une nécessité, cette acceptation n'est pas intégrée comme une volonté et un souhait de la part des représentants de l'éthique associative ; elle est au contraire plutôt rejetée symboliquement comme contraire aux vraies valeurs de l'associatif, lesquelles se concrétisent néanmoins par la présence et la gouvernance réaffirmée des bénévoles au sein du club.

D'un autre côté en effet, ils doivent aussi et surtout demeurer les "gardiens » des valeurs authentiques du sport associatif « légitime», sous peine de perdre les avantages symboliques associés à la promotion des valeurs sociales et culturelles de la cause associative sportive. De là découle leur posture ambivalente vis-à-vis des acteurs rémunérés dans le club sportif : si ces derniers sont nécessaires pour répondre de manière adéquate aux enjeux et aux mutations de l'offre sportive, ils représentent aussi les acteurs par lesquels s'immiscent des logiques économiques et «professionnelles» potentiellement menaçantes 
pour l'identité et les valeurs structurelles des clubs sportifs (Faure, Suaud, 2004). D'où le « travail» des dirigeants bénévoles pour contenir le développement des formes d'encadrement rémunéré en redéfinissant l'identité et les conditions du travail salarié à travers des valeurs et des modalités de fonctionnement historiquement constituées. Ils sont pour cela aidés par un contexte propre à renforcer une domination d'autant plus effective qu'elle se structure sur une fragilisation de la posture de salarié.

\section{De la confusion entre travail bénévole et travail rémunéré...}

Pour les bénévoles, la définition de l'activité d'encadrement dans un club sportif est basée sur le don. Cette idée revient sans cesse dans leurs propos et ne concerne pas seulement le statut de bénévole, mais s'adresse à tous ceux qui sont impliqués, d'une manière ou d'une autre, dans la vie du club.

L'un des aspects du travail dans un club sportif consiste donc en l'effacement des frontières entre travail bénévole et travail salarié dans le sens où, quel que soit le statut, cet impératif du « don » s'impose de fait à tous. Les « règles » du travail salarié sont définies à l'aune des valeurs et modes de fonctionnement bénévole, indépendamment de l'existence d'un cadre législatif: "Les horaires, c'est sur le contrat de travail (...). Il faut effacer au maximum l'aspect professionnel (...). L'important, si on veut que ça marche, et avec ou sans convention collective, il y a une règle: le professionnel, qui est censé avoir les compétences, il doit être bénévole dans l'esprit, et le bénévole, lui, qui est censé ne pas avoir les compétences, il doit être professionnel dans son travail. A partir de là, qu 'il soit bénévole ou professionnel, il n'y a pas de problèmes, ça fonctionne très bien. S'il n'y a pas ça, on va au devant des problèmes énormes. " (Président du club de gymnastique N)

Ainsi les dirigeants bénévoles (et employeurs) maintiennent d'autant mieux cette confusion entre bénévoles et salariés qu'ils engagent tacitement ces derniers à justifier leur rémunération par une disponibilité sans faille : «Si on a un professionnel qui arrive toujours en retard alors que le bénévole, lui... et bien il se dit "et attend, là, celui-là on le paye et moi je viens là bénévolement et je rame alors que lui il n'est pas là... le jour où il y a une compétition de championnat de France et tout"... le bénévole il vient et le salarié il ne vient pas pour donner un coup de main! Les bénévoles n'accepteront jamais. " (Président du club de gymnastique P). Dans un tel contexte, le fait de percevoir une rémunération est plutôt un handicap qui se heurte au principe du don gratuit et désintéressé qui prévaut dans le monde associatif sportif. Au lieu d'émanciper le salarié, il le contraint plutôt à adopter la posture qui convient pour prouver aux bénévoles que, en dépit de son salaire, il est capable d'actes gratuits. Car pour certains bénévoles, travailler dans un club sportif est une chance, un privilège, qui doit être considéré comme tel par le salarié. "C'est un des problèmes de l'association, c'est que l'employeur n'est jamais derrière eux. Il n'a pas le temps d'être derrière eux. Il n'y a pas de pointeuses. Les salariés sont un peu privilégiés. » (Président du club de football $\mathrm{G})$. De ce fait, pour les dirigeants bénévoles, le seul fait d'avoir procuré un emploi demeure un argument d'autosatisfaction, même s'ils ne se préoccupent pas des perspectives de carrière du salarié. Car le «manque de moyen» est effectivement un élément récurrent invoqué par les dirigeants bénévoles pour placer les salariés dans une situation d'incertitude quand aux perspectives d'évolution de leurs carrières. Il permet également aux bénévoles de tenir un discours décomplexé sur la nécessité imposée aux salariés de «créer » leurs emplois pour que ceux-ci continuent à exister.

\section{... au maintien du pouvoir des bénévoles}

Mais si les frontières entre travail bénévole et travail salarié tendent à être effacées sur le terrain de l'implication souhaitée, elles sont réhabilitées sur le terrain du statut, et des prérogatives qui lui sont associées, notamment en termes de pouvoir. Si les salariés sont sommés de montrer, à l'instar de l'exemple bénévole, leur «bonne volonté » en n'étant pas comptables de leurs heures et de leur rémunération, ils ne sont pas pour autant placés à égalité des bénévoles sur l'échiquier du club sportif. "Les salariés sont impliqués dans la vie du club, et assistent à tous les conseils d'administration. Bon s'il y a un vote délicat relatif à un cas institutionnel précis, ils ne votent pas, mais pour le fonctionnement courant, ils 
votent » (président du club de gymnastique N). Plus encore, dans certains cas, une participation trop active est perçue comme une menace par des dirigeants associatifs soucieux de garder leur pouvoir et enclins à percevoir dans l'implication (pourtant exigée) des salariés une tentative et une possibilité d'avoir la mainmise sur le club: "Parce que le salarié, dans des petites structures comme la nôtre, il peut faire exploser le président... si on n'y fait pas gaffe, le professionnel qui est là tout le temps, il peut toucher 600 personnes, alors que les bénévoles eux sont là de façon épisodique. Le pro a beaucoup de pouvoir... il y a beaucoup de structures qui explosent comme ça. » (Président du club de tennis E)

Les bénévoles développent donc un discours ambivalent vis-à-vis de l'implication des salariés : d'un côté, ils exigent une participation «bénévole » à la vie du club et, de l'autre, ils ne souhaitent pas qu'elle s'accompagne des prérogatives habituellement associées à un degré d'implication élevé. Dans le même registre, les bénévoles demandent aux salariés « d'être autonomes" dans leurs démarches et leurs initiatives, notamment pour alléger la charge des dirigeants du club, mais en même temps, ils veulent "garder la main» en exigeant d'être informés au plus près : «Au départ je leur demande... il serait inadmissible qu'il se passe des choses... je ne sais pas moi... des choses dans le club dont je ne sois pas au courant $»$. (Président du club de football I).

Ainsi, la posture des bénévoles place les salariés dans une situation problématique. Sommés de s'impliquer fortement dans la vie du club afin de justifier leur rémunération et d'effacer des différences mal perçues par les bénévoles, les salariés sont néanmoins maintenus dans des positions de subalternes dans la structure du pouvoir de l'association et sans cesse rappelés à leur instabilité professionnelle, alors même qu'ils se doivent de reconnaître leur «chance» de travailler dans le secteur associatif sportif.

Mais comment les acteurs salariés eux-mêmes vivent-ils leur condition au sein des clubs sportifs?

\section{LA VOCATION AU DÉTRIMENT DE L'ÉVOLUTION PROFESSIONNELLE}

Plusieurs travaux (Fleuriel, Chevalier, 2008; Hely, 2008) ont récemment mis en évidence la difficile normalisation du travail associatif au regard d'indicateurs tels que le respect du volume horaire contractualisé, le niveau de rémunération, la valorisation ou encore les perspectives d'évolution de carrière. Effectivement, notre enquête auprès des salariés de clubs sportifs marnais confirme ces données : souspayés, les salariés travaillent très fréquemment bien au-delà du volume horaire hebdomadaire indiqué sur leur contrat; ils sont soumis à une précarisation forte de l'emploi (notamment les entraîneurs salariés), et les clubs échantillonnés (les plus importants du département dans leur domaine sportif respectif) sont structurés de telle façon qu'ils n'ont quasiment aucune perspective d'évolution de carrière à leur proposer. De plus, conscient de l'inévitable précarité du travail rémunéré dans les clubs sportifs, les salariés se définissent comme responsables de la pérennité (ou non) de leur emploi et donc de la nécessité, pour eux, de s'engager pleinement et sans réserve au service du club pour préserver leur poste. En même temps, les propos de ces salariés traduisent souvent leur satisfaction de travailler dans le domaine sportif et de contribuer activement au développement de leur club. Ils reprennent à leur propre compte cette posture qui fait de l'engagement, de la disponibilité, du désintéressement et du dévouement des caractéristiques dominantes inhérentes au travail associatif sportif. Cette ambivalence peut se comprendre au regard des travaux de Norbert Alter (2002) autour de la relation de réciprocité qui écarte toute perspective en termes d'individu calculateur et rationalisant ses intérêts au sens économique du terme. Si les salariés du sport associatif «donnent» de leur temps et de leur personne, c'est parce qu'ils se sentent gratifiés par une relation de réciprocité qui apporte une légitimité et un sens au «don» qui les caractérise. Car «ce qui se joue dans l'échange social n'est pas une partie, un "enjeu", un avantage économique ou même symbolique. C'est, bien plus fondamentalement, la relation même qui unit les partenaires de l'échange, l'existence de la relation, et non l'avantage que l'on en retire. Cela explique les raisons 
pour lesquelles, pour préserver la qualité d'une relation, bon nombre d'acteurs acceptent de ne pas tirer avantage de leur situation, ou de leur pouvoir " (Alter, 2002, p. 275).

\section{Le métier comme engagement}

Comme nous l'avons montré précédemment, la gouvernance de la logique bénévole sur le champ associatif sportif valorise fortement les valeurs morales par lesquelles les bénévoles se définissent et se reconnaissent entre eux. De ce fait, dans ce contexte marqué par la cohabitation entre bénévoles et salariés, le capital associatif ${ }^{6}$ légitime des uns et des autres est logiquement structuré par des dispositions similaires telles que la disponibilité, le désintéressement, la gratuité de l'engagement.

Objectivement, rien n'oblige le salarié à faire sienne la logique bénévole en se « dévouant» pour le club. Pourtant, tous ceux qui ont été interrogés déclarent faire plus d'heures que le volume horaire inscrit sur leur contrat de travail : "Alors moi j'ai 35 heures de part la mairie, mais j'en fais plus que ça. Le lundi déjà je suis là de $8 H$ à $12 H$, l'après-midi je fais un peu de papier et tout ça, et je ne repars pas avant 19 heures, le mardi c'est pareil...» (Agent d'entretien et entraîneur dans le club de H). Mais cela ne semble leur poser aucun problème dans la mesure où dans tous les cas considérés, les salariés considèrent que la part de «bénévolat » qui leur est tacitement demandée par leurs employeurs fait partie intégrante du travail dans un club sportif; ils définissent donc leur métier à l'aune des catégories de l'engagement bénévole en se justifiant par un attachement à «l'esprit club». "Quand on est salarié dans une association sportive, il faut accepter le fait que l'on puisse en faire un peu plus. Du fait que l'on est confronté à des bénévoles qui eux donnent de leur temps en dehors de leur vie familiale ou profession-

\footnotetext{
${ }^{6}$ Le « capital associatif » peut être considéré comme l'ensemble des propriétés qui permettent au bénévole d'exister « associativement » dans cet espace, c'est-à-dire d'être reconnu par lui (Walter, 2001). La notion de «capital associatif» est donc ici comprise comme « capital spécifique » (Bourdieu, 1980) qui n'a de valeur que référée à un type d'espace associatif et à sa logique immanente. Nous rappelons ici, en effet, qu'un capital ou une espèce de capital, c'est ce qui est efficient dans un champ déterminé, à la fois en tant qu'arme et en tant qu'enjeu de lutte, « ce qui permet à son détenteur d'y exercer un pouvoir, une influence, donc d'y exister » (Bourdieu, 1996).
}

nelle. Moi c'est mon éthique personnelle, j'estime devoir leur rendre ça aussi et leur en donner par rapport à ça» (salariée dans l'association de gymnastique $\mathrm{H}$ ). Cette posture peut être mise en relation avec les trajectoires des salariés considérés, dont la plupart évoluent depuis leur plus jeune âge dans le cadre du sport associatif. Souvent issus du club, les salariés sont bien connus de leurs employeurs avant même d'être recrutés et ont souvent un parcours préalable en tant que bénévole. Le mode de recrutement des salariés et leurs modalités de travail montrent effectivement que les salariés sont d'abord liés au club sur la base de leur appartenance à une communauté morale. La plupart d'entre eux nourrissent un attachement affectif fort à «leur sport» et/ou au club dans lequel ils travaillent, et ce d'autant plus qu'ils considèrent le club sportif comme un environnement privilégié, « protégé » de la violence « ordinaire » du monde du travail par un tissu d'échanges fondés prioritairement sur des relations affectives et affinitaires. « $L a$ différence elle se fait entre les domaines associatif et l'entreprise, là c'est clair, si on a un gros souci, je pense que les membres du comité ne nous laisseraient pas dans la merde (...) il y a plus d'humanité (...) même si on se plaint parfois, je pense qu'il faut garder les pieds sur terre et se dire qu'on est quand même bien lotis par rapport à d'autres » (Salariée dans le club de gymnastique $\mathrm{M}$ ).

Les discours produits traduisent ainsi une forme de gratitude vis-à-vis du club et/ ou de dirigeants bénévoles qui jouent ou ont joué un rôle essentiel dans la trajectoire du salarié, le motivant à « rendre » ce dont il estime avoir bénéficié à son avantage. Le surinvestissement dans une activité donnée montre que « tout en étant inscrite dans une relation salariée, leur situation de travail est pénétrée par une économie du don et du contre-don propre à l'univers sportif » (Chimot, Schotté, 2006, p. 5). Ainsi le salarié est prédisposé par sa trajectoire et son capital associatif à définir prioritairement son métier à travers les catégories de l'engagement « gratuit 》 et « dévoué » afin de rester dans une forme d'échange gouverné par les logiques du bénévolat. Mais il en retire un profit symbolique qui le conforte dans une identité sociale à travers laquelle il est reconnu et il se reconnaît dans une relation de réciprocité (Alter, 2002) structurée 
par l'histoire du salarié. "Surtout qu'en plus moi je suis vraiment issu de ce monde là, et c'est quelque chose de vraiment important (...) j'ai été bénévole dans cette association jusqu'à 17-18 ans (...) moi je pense que les salariés c'est utile, c'est nécessaire, mais il faudrait des gardes fous, qu'on reste quand même dans un monde principalement géré par des bénévoles " (Salarié dans l'association de gymnastique R). Pour le salarié intégré dans un système d'échange collectif, "ce qui compte n'est pas la nature ou la valeur marchande de la chose échangée, mais le fait qu'elle crée un lien social fort, intégrant des dimensions à la fois symboliques, matérielles et affectives entre les contrôleurs et les contrôlés" (Alter, 2002, p. 269).

\section{Le métier comme passion}

Si l'effacement de la frontière entre bénévole et salarié est au principe d'une propension à définir le métier comme un « engagement», l'appartenance au champ sportif structure chez le salarié une disposition très marquée à effacer l'idée de «métier» derrière celle de « passion ». Car les salariés évoluent dans un espace qui valorise fortement un continuum entre une discipline sportive investie et l'emploi occupé. Plus précisément, le recours à la «passion » est souvent mobilisé pour justifier l'adhésion à une entière disponibilité au sein du club, parfois au détriment de la vie privée.

Mais ces «sacrifices » pleinement acceptés ne sont pas évoqués comme une oppression, ni même comme une contrainte ; ils sont au contraire présentés comme une des conditions permettant d'évoluer dans un univers socialement convoité : «je travaille du lundi au dimanche, donc je fais à peu près... enfin je compte même pas, c'est l'enfer, là... mais bon c'est une passion, donc c'est pas une contrainte, à savoir qu'on a quand même la chance de baigner dans un milieu où on est... bien, quoi, bon c'est quand même du loisir, c'est quand même un jeu, au départ c'est du jeu, donc il y a une bonne humeur, il y a une bonne ambiance. " (moniteur possédant le brevet d'État, club de tennis F)

Comme le soulignent C. Chimot et M. Schotté (2006, p. 4), « si la passion sportive pèse, ce n'est pas seulement en tant que pré-requis à l'entrée dans l'espace fédéral. C'est aussi parce qu'elle vient donner une forme particulière aux activités de travail ». Ainsi la chance de travailler dans le sport est liée au caractère « aventurier» d'un métier où «tout est sans cesse remis en cause » et où l'instabilité du poste donne de la valeur à une existence professionnelle risquée. Pour ces salariés, le «fonctionnariat » (entraîneur de football dans le club $\mathrm{G}$ ) représente l'antithèse de leur métier et symbolise ce qu'ils rejettent en raison de son incompatibilité avec l'idée du « risque » et de la «remise en question» valorisée dans le sport. «Dans la compétition, la recherche de performance, même amateur, il est logique que l'on ne tombe pas sur un fonctionnariat. C'est le côté qui m'excite. C'est souvent au détriment de l'entraîneur parce que l'on ne renouvelle pas son contrat mais cela peut permettre aussi de repartir et de quitter son contrat » (entraîneur de football dans le club G). Dit autrement, c'est l'adhésion aux logiques de la "passion sportive» qui semble définir les contours de l'employabilité du salarié jugé en fonction de sa capacité à intégrer une "culture de l'incertitude permanente » qui normalise la responsabilité individuelle du salarié dans la dynamique de son propre emploi. "Je suis responsable de mon truc, voilà, quoi, c'est moi qui fait mon emploi du temps, c'est moi qui doit m'arranger pour proposer des activités qui plaisent, pour que des gens viennent, pour que les gens qui sont là restent... je gère tout moi-même, $q u o i »$ (Monitrice de fitness dans le club de gymnastique $\mathrm{N}$ ).

\section{La valeur du capital sportif}

La définition du métier comme «passion» renvoie au fait que le salarié ancre son discours et la représentation de son activité professionnelle avant tout dans l'imaginaire de l'univers sportif. Les salariés associent pleinement les valeurs du sport à leur métier, en soulignant à la fois l'importance du rôle social, voire éducatif du club, et de leur mission et, plus particulièrement pour les entraîneurs, l'aléa et l'incertitude de la compétition.

Or l'analyse des dispositions sociales, et notamment du capital sportif, des salariés interviewés montre un rapport entre le volume de capital sportif et les stratégies d'émancipation et de moindre dépendance aux dirigeants bénévoles. Ainsi plus un entraîneur ou un moniteur peut se prévaloir d'un niveau sportif 
présent ou passé avantageux, plus ses possibilités de négociation, et donc d'échange, avec les dirigeants du club sont élevées. Il peut notamment mettre en jeu ses facilités pour trouver un travail dans un autre club dans le cas où ses conditions ne seraient pas prises en compte. Il peut aussi revendiquer plus aisément sur le terrain du salaire et d'une autonomie dans le travail. En effet, la valeur de son capital sportif lui confère une expertise reconnue qui lui donne un pouvoir en matière de travail et d'investissement dans le club et la possibilité de négocier favorablement ses propres conditions aux dirigeants bénévoles. "Moi ça se passe bien, je suis dirigé sans être dirigé, parce que je n'ai pas l'impression qu'il m'impose quoi que ce soit, je prends les terrains que je veux, je fais mon emploi du temps comme je veux » (moniteur de tennis $\mathrm{BE} /$ responsable du centre d'entrainement «élite » du club de tennis A). Dans ce cas, le capital sportif agit comme une plus-value qui assure au moniteur salarié une reconnaissance de ses compétences de professionnel par les dirigeants bénévoles. Mais si ce capital assure au salarié concerné une indépendance apparemment plus importante que celle dont jouissent ceux qui ne peuvent pas se prévaloir des mêmes dispositions sportives, il demeure fragile car fortement indexé aux aléas de la vie sportive et associative du club. Car l'évaluation du travail produit est liée sommairement aux résultats en compétition, par définition aléatoires et incertains. De plus, les projets de ce salarié restent dépendants de la politique sportive du club. En cas de changement, ses dispositions a priori favorables peuvent rapidement devenir défavorables, notamment si l'axe compétitif perd son statut dominant et structurant au sein du club. situer en décalage avec la culture associative dominante. Le discours traduisant une émancipation à l'égard de l'éthos bénévole se heurte aux jeux et enjeux de pouvoirs au sein de l'association, l'espace associatif restant dominé par les bénévoles (Loirand, 2001 ; Fleuriel, Schotté, 2008). Ainsi, le salarié qui n'adopte pas cette logique bénévole se retrouve dans une position délicate et en marge du système dominant. Car les dirigeants bénévoles attendent avant tout du salarié une posture traduisant sa capacité à s'engager pour le bien collectif du club et dans l'intérêt général qu'ils représentent de manière emblématique. Cette posture, dont l'enjeu est la reconnaissance et l'existence légitime au sein du club, est favorisée par la porosité entre carrière professionnelle et engagement pour une cause (Chimot, Schotté, 2006); cela suppose implicitement que le salarié renonce à privilégier un intérêt professionnel sur l'intérêt vocationnel qui définit la forme dominante du travail au sein du club sportif. Pour autant, si les salariés du sport associatif « donnent» de leur temps et de leur personne, c'est parce qu'ils évoluent dans cette relation de réciprocité à travers laquelle ils se réapproprient la dimension symbolique qui donne sens à leur engagement au sein du club. «Ce qui importe, dans ce type de relation, est bien plus de savoir que l'on peut compter sur l'autre - parce qu'on l'a obligé en lui donnant quelque chose - que le fait de connaître le moment du retour» (Alter, 2002, p. 267). Le don s'intègre dans un système d'échange au sein de l'action collective et donne existence aux identités individuelles. "Si l'individu était étroitement calculateur, il n'aurait aucun intérêt à participer à l'action collective, laquelle n'existerait pas » (Alter, 2002, p. 266). Il s'agit ici d'un «phénomène social total» (Mauss, 1950) où le fait de donner oblige l'autre à donner à son tour. L'adhésion précoce à un champ associatif sportif construit sur la légitimité d'une forme d'échange non économique prédispose notamment ces acteurs à «investir une relation plus qu'ils n'investissent dans une relation » (Alter, 2002, p. 267). De ce point de vue, les acteurs sont d'autant plus prédisposés à «entrer dans le jeu» que leurs trajectoires sont marquées par une adhésion précoce à un champ associatif sportif historiquement construit sur la base d'un déni du travail rémunéré (Fleuriel, 2004). Ceux qui sont les plus disposés à exister dans l'espace d'un club sportif possèdent donc les propriétés sociales et symboliques nécessaires pour investir favorablement la relation de réciprocité, donner sens à leur action et en retirer un intérêt au sens «'illusio »?

\footnotetext{
${ }^{7}$ «L'illusio, c'est le fait d'être pris au jeu, d'être pris par le jeu, de croire que le jeu en vaut la chandelle, ou, pour dire les choses simplement, que ça vaut la peine de jouer » (Bourdieu, 1996).
} 
Alter N. (2002), «Théorie du don et sociologie du monde du travail », La revue du MAUSS, $\mathrm{n}^{\circ} 20$, pp. 263-285.

Augustin J.-P. (2003), Le sport et ses métiers : nouvelles pratiques et enjeux d'une professionnalisation, Paris, La Découverte.

Bourdieu P. (1998), «Comment peut-on être sportif?», in Questions de sociologie, Paris, Les Éditions de Minuit, pp. 173-195.

Bourdieu P. (1996), Raisons pratiques. Sur la théorie de l'action, Paris, Points, collection « Essai ».

Bourdieu P. (1980). Le sens pratique, Paris, Éditions de Minuit.

Chanial P. (1998), «La délicate essence de la démocratie : solidarité, don et association », La revue du MAUSS, $\mathrm{n}^{\circ} 11$, pp. 28-43.

Chantelat P. (2001) (sous la dir.), La professionnalisation des organisations sportives, nouveaux enjeux, nouveaux débats, Paris, L'Harmattan.

Chimot C., Schotté M. (2006), « Travailler dans une organisation sportive. Entre engagement passionné et investissement professionnel », Regards Sociologiques, $\mathrm{n}^{\circ} 32$, pp. 61-78.

Falcoz M., Walter E. (2007), « Travailler dans un monde de bénévoles : contraintes et limites de la professionnalisation dans les clubs sportifs », RECMA, n 306, pp. 78-91.

Faure J.-M., Suaud C. (2004), Pour une sociologie des espaces sportifs nationaux. Actes du premier congrès de la Société française de sociologie du sport, université de Toulouse 3 Paul Sabatier, pp. 5-31.

Fleuriel S. (2006), «Le travail dénié et les jeux olympiques: entre passion et intérêts », Société contemporaines $\mathrm{n}^{\circ} 63$, Les presses de Science Po, pp. 85-103.
Fleuriel S. (2004), Le sport de haut-niveau en France, Grenoble, PUG.

Fleuriel S., Chevalier V. (2008), « Travail bénévole et marché du travail sportif », Les Mondes du travail, $\mathrm{n}^{\circ} 5$, pp. 67-79.

Fleuriel S., Schotté M. (2008), Sportifs en danger. La condition des travailleurs sportifs, Bellecombe-enBauges, Éditions du Croquant.

Hely M. (2008), «A travail égal, salaire inégal : ce que travailler dans le secteur associatif veut dire», Sociétés contemporaines, $\mathrm{n}^{\circ}$ 69, pp. 125-148.

Hely M. (2004), «Les différentes formes d'entreprises associatives", Sociologies pratiques, $\mathrm{n}^{\circ} 9$, pp. 27-51.

Observatoire national des métiers de l'animation et du sport (2006), Ithaque, Enquête auprès des entreprises de la branche sport, Paris.

Legay A. (2001), «La professionnalisation de l'emploi associatif; l'exemple des permanents des associations intermédiaires », document, $\mathrm{n}^{\circ} 158$, série " Observatoire », Céreq.

Le Roux N. (2006), «Évolution des connaissances et perspectives de recherche sur l'emploi et la professionnalisation dans le secteur du sport» in P. Bouchet et C. Pigeassou (sous la dir.), Management du sport : actualités, développements et orientations pour la recherche, Montpellier, AFRAPS, pp. 113-141.

Le Roux N., Aguettant N. (2006), «L'emploi sportif en France et ses évolutions : quel état des lieux ? », in J.-P. Augustin (sous la dir.), Vers les métiers de l'animation et du sport : la transition professionnelle, Paris, La Documentation française, pp. 147-165.

Liotard P. (2004), «L'éthique sportive : une morale de la soumission?» in M. Attali (sous la dir.), Le sport et ses valeurs, Paris, La Dispute, pp. 117-156. 
Loirand G. (2004), « Professionnalisation : de quoi parle-t-on?» in Société de Sociologie du Sport de Langue Française (Ed.), Dispositions et pratiques sportives. Débats actuels en sociologie du sport, Paris, l'Harmattan, pp. 217-227.

Loirand G. (2003), « Les paradoxes de la "professionnalisation" des associations sportives $»$ in L. Prouteau (sous la dir.), Les associations entre bénévolat et logiques d'entreprise, Rennes, PUR, pp. 85-103.

Loirand G. (2001), « Le bénévolat sportif : les ambiguïtés d'un engagement » in P. Chantelat (sous la dir.), La professionnalisation des organisations sportives, nouveaux enjeux, nouveaux débats, Paris, L'Harmattan, pp. 273-300.
Marchal E. (1992), «L'entreprise associative entre calcul économique et désintéressement», Revue Française de Sociologie, vol. 3, n 33, pp. 365-390.

Mauss M. (1950), Sociologie et anthropologie, Presses universitaires de France.

Tchernonog V. (2007), Le paysage associatif français - Mesures et évolutions, Paris, Juris associations Dalloz.

Walter E. (2001), Logiques de l'engagement dans le sport associatif. De l'illusio bénévole. Thèse de doctorat STAPS mention «Sport et sciences sociales », université Marc Bloch de Strasbourg.

\section{Résumé}

\section{Être salarié dans un club sportif : une posture problématique}

\section{Marc Falcoz et Emmanuelle Walter}

Les enjeux du sport associatif français s'inscrivent dans un contexte de développement significatif de l'emploi dans le secteur associatif en général. Mais si le nombre de professionnels de l'encadrement associatif sportif a fortement augmenté depuis une vingtaine d'année, il ne remet pas en cause la primauté du bénévolat dans ce secteur. De ce fait, exister professionnellement dans ce monde de bénévoles se révèle problématique: recrutés sur la base de compétences qualifiées par la possession du diplôme et/ou brevet adéquat (condition sine qua non en France pour encadrer sportivement contre rémunération), les salariés sont avant tout reconnus et distingués dans le club sportif par leurs dispositions au don de soi et leur perméabilité aux injonctions morales de leurs employeurs bénévoles.

\section{Mots clés}

Professionnalisation, association, bénévolat, salarié, culture-loisir-sport. Journal of Economic Literature: J 44 